\title{
Apolipoprotein A-I stimulates AMP-activated protein kinase and improves glucose metabolism
}

\author{
R. Han $\cdot$ R. Lai $\cdot$ Q. Ding $\cdot$ Z. Wang $\cdot$ X. Luo $\cdot$ \\ Y. Zhang $\cdot$ G. Cui $\cdot$ J. He $\cdot$ W. Liu $\cdot$ Y. Chen
}

Received: 21 May 2007 / Accepted: 31 May 2007 / Published online: 18 July 2007

(C) Springer-Verlag 2007

\begin{abstract}
Aims/hypothesis In humans, one of the hallmarks of type 2 diabetes is a reduced plasma concentration of HDL and its major protein component, apolipoprotein A-I (APOA-I). However, it is unknown whether APOA-I directly protects against diabetes. The aim of this study was to characterise the functional role of APOA-I in glucose homeostasis.

Methods The effects of APOA-I on phosphorylation of AMPactivated protein kinase (AMPK) and acetyl-coenzyme A carboxylase (ACC), glucose uptake and endocytosis were analysed in $\mathrm{C} 2 \mathrm{C} 12$ myocytes. Glucose metabolism was investigated in Apoa-I knockout (Apoa- $\Gamma^{\prime-}$ ) mice.

Results APOA-I was able to stimulate the phosphorylation of AMPK and ACC, and elevated glucose uptake in $\mathrm{C} 2 \mathrm{C} 12$ myocytes. APOA-I could be endocytosed into $\mathrm{C} 2 \mathrm{C} 12$ myotubes through a clathrin-dependent endocytotic process. Inhibition of endocytosis abrogated APOA-I-stimulated AMPK phosphorylation. In Apoa- $\Gamma^{--}$mice, AMPK phosphorylation was reduced in skeletal muscle and liver, and expression of gluconeogenic enzymes was increased in liver. In addition, the $A p o a-\Gamma^{/-}$mice had increased fat content and compromised glucose tolerance.
\end{abstract}

R. Han $\cdot$ Q. Ding $\cdot$ Z. Wang $\cdot$ X. Luo $\cdot$ Y. Zhang $\cdot$ G. Cui $\cdot$ J. He $\cdot$

W. Liu $\cdot$ Y. Chen $(\bowtie)$

Institute for Nutritional Sciences, Shanghai Institutes

for Biological Sciences, Chinese Academy of Sciences,

Graduate School of the Chinese Academy of Sciences,

Shanghai 200031, China

e-mail: ychen3@sibs.ac.cn

R. Lai

Department of Biochemistry and Molecular Biology,

Gannan Medical College,

Ganzhou, Jiangxi 341000, China
Conclusions/interpretation Our data indicate that APOA-I has a protective effect against diabetes via activation of AMPK. ApoA-I deletion in the mouse led to increased fat mass and impaired glucose tolerance.

Keywords AMP-activated protein kinase - AMPK . Apolipoprotein A-I · APOA-I · Endocytosis · Fat mass · Glucose homeostasis · Insulin resistance · Type 2 diabetes

$\begin{array}{ll}\text { Abbreviations } \\ \text { ABCA1 } & \text { ATP-binding cassette transporter A1 } \\ \text { ACC } & \text { acetyl-coenzyme A carboxylase } \\ \text { AMPK } & \text { AMP-activated protein kinase } \\ \text { APOA-I } & \begin{array}{l}\text { apolipoprotein A-I } \\ \text { apolipoprotein B }\end{array} \\ \text { APOB } & \text { endothelial nitric oxide synthase } \\ \text { eNOS } & \text { glucose-6-phosphatase } \\ \text { G6Pase } & \begin{array}{l}\text { impaired glucose tolerance } \\ \text { IGT }\end{array} \\ \text { M- } \beta C D & \text { methyl } \beta \text {-cyclodextrin } \\ \text { PEPCK } & \text { phosphoenolpyruvate carboxykinase } \\ \text { RIPA } & \text { Radioimmunoprecipitation assay } \\ \text { SR-BI } & \text { scavenger receptor class B type I } \\ \text { SR-BII } & \text { scavenger receptor class B type II }\end{array}$

\section{Introduction}

Apolipoprotein A-I (APOA-I) is the most abundant protein constituent of HDL [1]. Extensive studies have demonstrated that HDL and APOA-I are both able to reduce the risk of atherosclerosis, the leading cause of death in industrialised countries [2]. APOA-I is critical to HDL function in reverse cholesterol transport, the delivery of cholesterol from peripheral tissue to the liver for catabolism and steroidogenesis [1]. APOA-I is involved at 
various stages in this process: in the transfer of free cholesterol by ATP-binding cassette transporter A1 (ABCA1) to acceptors, and in their subsequent esterification by HDL-associated enzyme lecithin cholesterol acyl transferase (LCAT) to form cholesteryl esters [3]. APOA-I is also involved in the delivery of cholesteryl esters to the liver via scavenger receptor class B type I (SR-BI) [4]. A decreased bioavailability of endothelium-derived NO is an important antecedent to atherosclerosis, and it was recently reported that APOA-I could upregulate the activity of endothelial nitric oxide synthase (eNOS), likely through specific phosphorylation of AMP-activated protein kinase (AMPK) in endothelial cells [5]. However, the intracellular function of APOA-I is largely uncharacterised.

In addition to their anti-atherogenic function, HDL and APOA-I are inversely correlated to the development of type 2 diabetes. In humans, one of the critical diagnostic markers of insulin resistance or metabolic syndrome is reduced plasma concentrations of HDL cholesterol and APOA-I [69]. Patients with type 2 diabetes are known to have low levels of APOA-I attributable to the increased catabolism of APOA-I and its decreased synthesis [10]. On the other hand, an increase in glucose concentration has been shown to cause downregulation of Apoa-I gene transcription [11]. It was recently reported that a mutated AA genotype of the Apoa-I MspI polymorphism conferred a greater risk of IGT and type 2 diabetes [12]. However, it remains unclear at present whether HDL and/or APOA-I directly protect against diabetes, and if so, what the underlying molecular mechanism is.

\section{Methods}

Materials All tissue culture supplies were obtained from Gibco BRL (Grand Island, NY, USA). The antibodies for AMPK, phosphorylated AMPK, ACC and phosphorylated ACC were from Cell Signaling (Beverly, MA, USA). The purified APOA-I was from Fluka (St Gallen, Switzerland), apolipoprotein B (APOB) was from Fitzgerald (MA, USA), and BSA was from Sigma (St Louis, MO, USA). Caveolin-1 antibody was from Abcam (Cambridge, UK). Recombinant human adiponectin and globular adiponectin were from R\&D Systems (Minneapolis, MN, USA).

Cell culture C2C12 cells were maintained in DMEM supplemented with $10 \%$ fetal bovine serum (FBS). Differentiation of $\mathrm{C} 2 \mathrm{C} 12$ cells was induced by culturing in $2 \%$ horse serum-containing DMEM changed daily for 5-7 days.

Western blotting analysis For western blotting analysis, the cells were lysed in radioimmunoprecipitation assay (RIPA) buffer (150 mmol/1 NaCl, 1\% [vol./vol.] Triton X-100,
$0.5 \%$ [wt/vol.] sodium deoxycholate, $0.1 \%$ [wt/vol.] SDS, $50 \mathrm{mmol} / 1$ Tris-HCl, $\mathrm{pH}$ 7.4) containing phosphatase inhibitors and a protease inhibitor cocktail (Sigma). The lysate was subjected to SDS-PAGE, transferred to poly (vinylidene fluoride) (PVDF) membranes, and incubated with the primary antibodies, followed by horseradish peroxidase-conjugated secondary antibody (Amersham, Little Chalfont, Bucks, UK). The bound antibody was visualised using enhanced chemiluminescence reagents (Pierce, Rockford, USA).

Glucose uptake assay Assessment of 2-[ $\left.{ }^{3} \mathrm{H}\right]$ deoxyglucose uptake was performed as described previously [13]. Briefly, differentiated $\mathrm{C} 2 \mathrm{C} 12$ myocytes were grown in six-well plates and were starved in DMEM containing $25 \mathrm{mmol} /$ 1 glucose for $5 \mathrm{~h}$. After rinsing with Krebs Ringer phosphate HEPES (KRPH) buffer (136 mmol/1 NaCl, $20 \mathrm{mmol} / \mathrm{l} \mathrm{HEPES}, 1 \mathrm{mmol} / 1 \mathrm{MgSO}_{4}, 1 \mathrm{mmol} / 1 \mathrm{CaCl}_{2}$, $4.7 \mathrm{mmol} / 1 \mathrm{KCl}, 5 \mathrm{mmol} / 1$ sodium phosphate buffer, $\mathrm{pH} 7.4$ ), the cells were incubated for $30 \mathrm{~min}$ at $37^{\circ} \mathrm{C}$ in the absence or presence of adiponectin, insulin or APOA-I. Glucose uptake was measured by incubating the cells in $50 \mathrm{nmol} / 1$ of $2-\left[{ }^{3} \mathrm{H}\right]$ deoxyglucose for $5 \mathrm{~min}$ at room temperature. Non-specific cell-associated radioactivity was measured by the addition of $10 \mu \mathrm{mol} / \mathrm{l}$ cytochalasin $\mathrm{B}$. Cells were washed three times with ice-cold PBS and lysed in $0.2 \mathrm{~mol} / 1 \mathrm{NaOH}$. Radioactivity was measured by liquid scintillation counting. Each condition was assayed in triplicate, and data are representative of more than three independent experiments.

Endocytosis analysis of APOA-I APOA-I was labelled using a Alexa Fluor 555 Microscale Protein labelling kit (Molecular Probes, Eugene, OR, USA) according to the manufacturer's instructions. Alexa Fluor 488-conjugated transferrin was purchased from Molecular Probes. For colocalisation of transferrin and APOA-I, differentiated $\mathrm{C} 2 \mathrm{C} 12$ cells were serum-starved for $2 \mathrm{~h}$ and then incubated with $50 \mu \mathrm{g} / \mathrm{ml}$ transferrin and $10 \mu \mathrm{g} / \mathrm{ml}$ labelled APOA-I in DMEM for $2 \mathrm{~h}$. The surface-bound transferrin and APOA-I were washed off with an ice-cold solution of $150 \mathrm{mmol} /$ $1 \mathrm{NaCl}$ and $20 \mathrm{mmol} / 1$ sodium citrate at $\mathrm{pH} 5.0$, three times. The cells were then fixed by $4 \%$ [vol./vol.] paraformaldehyde and imaged by confocal microscopy. (LSM 510; Zeiss, Oberkochen, Germany). For the potassium depletion experiment, $\mathrm{C} 2 \mathrm{C} 12$ cells were incubated in DMEM:water $(1: 1)$ at $37^{\circ} \mathrm{C}$ for $5 \mathrm{~min}$, followed by incubation in minimal medium (20 mmol/1 HEPES at pH 7.5, $140 \mathrm{mmol} / \mathrm{l} \mathrm{NaCl}$, $1 \mathrm{mmol} / 1 \mathrm{CaCl}_{2}, 1 \mathrm{mmol} / 1 \mathrm{MgSO}_{4}, 5.5 \mathrm{mmol} / 1$ glucose, $0.5 \%$ [wt/vol.] BSA) at $37^{\circ} \mathrm{C}$ for $1 \mathrm{~h}$. Cholesterol depletion, which affects caveolae-mediated endocytosis, was carried out by incubating the cells in $2.5 \mathrm{mmol} / \mathrm{l}$ methyl $\beta$ cyclodextrin (M- $\beta \mathrm{CD}$; Sigma) at $37^{\circ} \mathrm{C}$ for $1 \mathrm{~h}$. 
Animal studies Apoa-I Knockout (Apoa- $\Gamma^{1-}$ ) C57BL/6J mice were purchased from the Jackson Laboratory (Bar Harbor, ME, USA) [14]. All mice were given a standard chow diet $(4.5 \% \mathrm{wt} / \mathrm{wt}$ fat $)$ and had a regular $12 \mathrm{~h}$ dark/light cycle. Body fat content was determined by NMR using a Minispec mq10 NMR Analyzer (Bruker Optics, Billerica, MA, USA). Mice were killed at 12 weeks of age and the retroperitoneal fat pad was excised and weighed. For western blotting analysis, mice were killed at 12 weeks of age and the whole tissue extracts were prepared using RIPA buffer containing phosphatase inhibitors (Sigma) and a protease inhibitor cocktail (Sigma). The lysate was sonicated for $30 \mathrm{~s}$ and then centrifuged for $20 \mathrm{~min}$ at $20,000 \times \mathrm{g}$ to remove the cellular debris, and the supernatant fraction was used for western blotting analysis. For the glucose tolerance test, mice at 12 weeks of age were fasted for $3 \mathrm{~h}$ and then injected intraperitoneally with glucose $(2 \mathrm{~g} / \mathrm{kg}$ body weight). Blood glucose levels were monitored before and at 30,60 and $120 \mathrm{~min}$ after injection using a glucometer (FreeStyle; TheraSense, Alameda, CA, USA). The AUCs were determined using OriginPro software (OriginLab, Northampton, MA, USA). Serum insulin was measured with a commercially available LINCOplex Mouse Adipokine Immunoassay kit from Linco Research (St Charles, MO, USA). Transcripts of the genes encoding phosphoenolpyruvate carboxykinase (Pepck, also known as Pckl) and glucose-6-phosphatase (G6Pase, also known as G6pc) were determined by real-time RT-PCR, which was carried out in $25 \mu \mathrm{l}$ of reaction buffer with SYBR Green (Molecular Probes). All reactions were performed in triplicate in an iCycler iQ system (Bio-Rad, Hercules, CA, USA). To confirm amplification specificity, the PCR products from each primer pair were subjected to a melting curve analysis. The gene encoding $\beta$-actin $(A c t b)$ was used for internal normalisation. The primers used for the genes were (forward and reverse sequences are shown, respectively): 5'-GTCTATGAAGCCCTCAGCT-3' and 5'-AAG AAGGGTCGCATGGCAA-3' for PEPCK, 5'-GACTCCC AGGACTGGTTCAT-3' and 5'-GGGCGTTGTCCAAA CAGAAT-3' for G6Pase, 5'-GATCATTGCTCCTCCTGA GC-3' and 5'-ACTCCTGCTTGCTGATCCAC-3' for Actb.

Statistical analysis Multiple comparisons were analysed by ANOVA, and pair-wise comparison was by Student's $t$ test. Results are presented as means $\pm \mathrm{SD}$, and a $p$ value of $<0.05$ was considered statistically significant.

\section{Results}

Effect of APOA-I on phosphorylation of AMPK and ACC, and glucose uptake in C2C12 myotubes Previous studies with APOA-I have mainly focused on its extracellular cardioprotective actions [1]. To investigate the potential role APOA-I in energy and glucose metabolism, we analysed the functions of APOA-I in $\mathrm{C} 2 \mathrm{C} 12$ myocytes. In agreement with previous reports [15], treatment of $\mathrm{C} 2 \mathrm{C} 12$ myocytes with globular or full-length adiponectin caused the rapid stimulation of phosphorylation of Thr172 on the AMPK $\alpha$-subunit (Fig. 1a,b). Interestingly, treatment with APOA-I also caused a rapid increase in AMPK phosphorylation (Fig. 1a,b). However, treatment with both APOA-I and adiponectin did not appear to have a synergistic effect on AMPK phosphorylation (Fig. 1a,b). Treatment of the cells with APOB, the most abundant protein constituent of LDL, had no effect on AMPK phosphorylation (Fig. 1d,e). Treatment with BSA also had no effect on AMPK phosphorylation (Fig. 1d,e). As APOA-I mediates cholesterol efflux from cells, we analysed whether cholesterol efflux itself increases AMPK phosphorylation. M- $\beta C D$ was used to selectively extract cholesterol from the plasma membrane [16]. As shown in Fig. 1d, M- $\beta C D$ had no effect on AMPK phosphorylation. Furthermore, we observed that APOA-I increased AMPK phosphorylation in a dosedependent manner and reached a maximum 2 min after treatment, similar to the kinetics of adiponectin action (Fig. 1g). It is noteworthy that APOA-I at a concentration lower than the physiological range $(1-1.5 \mathrm{mg} / \mathrm{ml}[100$ $150 \mathrm{mg} / \mathrm{dl}]$ in serum) was sufficient to activate AMPK.

AMPK is involved in the regulation of fatty acid oxidation in skeletal muscle through phosphorylation (at Ser79) and inhibition of acetyl-coenzyme A carboxylase (ACC) $[17,18]$. As expected, treatment of $\mathrm{C} 2 \mathrm{C} 12$ myocytes with globular or full-length adiponectin increased ACC phosphorylation (Fig. 1a,c). Treatment with APOA-I also markedly elevated ACC phosphorylation (Fig. 1a,c). Consistent with their effects on AMPK phosphorylation, APOB, BSA and M- $\beta C D$ had no effect on ACC phosphorylation (Fig. 1d,f).

We next analysed the effect of APOA-I on glucose uptake in $\mathrm{C} 2 \mathrm{C} 12$ cells. APOA-I stimulated glucose uptake in these cells, as did the administration of adiponectin (Fig. 2a), which has been shown to regulate glucose uptake via activation of AMPK [17, 19]. Treatment with APOA-I and insulin had an apparent synergistic effect on glucose uptake (Fig. 2b), but APOA-I and adiponectin appeared to have no such synergistic effect (Fig. 2a.). In addition, stimulation of glucose uptake by APOA-I was increased with increasing concentrations of APOA-I (Fig. 2c). Collectively, these data suggest that APOA-I is able to regulate glucose uptake in skeletal muscle cells.

Endocytosis of APOA-I in C2C12 cells To provide direct evidence that APOA-I is able to exert its function intracellularly, we examined cellular uptake and trafficking of APOA-I. APOA-I was labelled with the fluorescent 
a

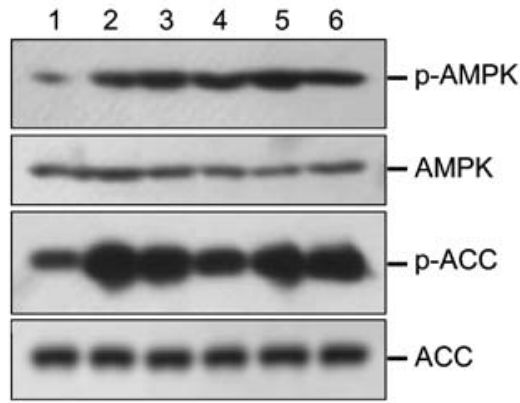

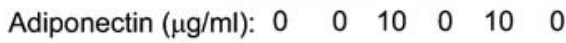

gAdiponectin $(\mu \mathrm{g} / \mathrm{ml}): 0 \begin{array}{llllll}0 & 0 & 0 & 2.5 & 0 & 2.5\end{array}$

ApoA-I $(\mu \mathrm{g} / \mathrm{ml}): 0 \quad 20 \quad 0 \quad 0 \quad 20 \quad 20$

d

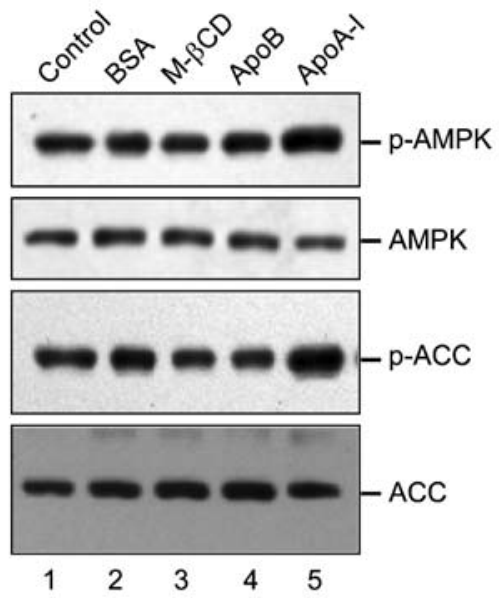

b

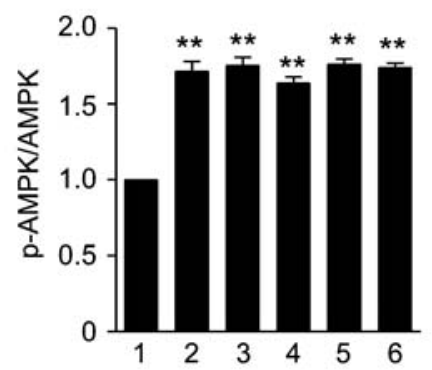

e

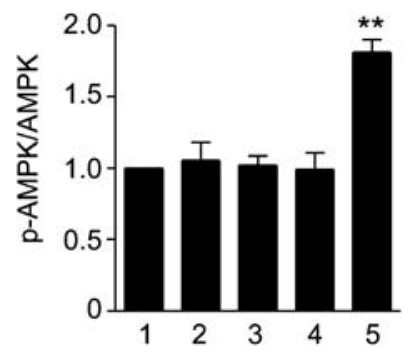

C

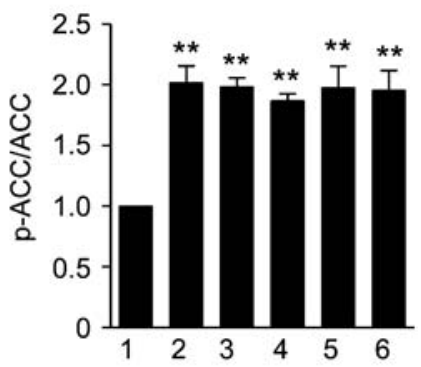

f

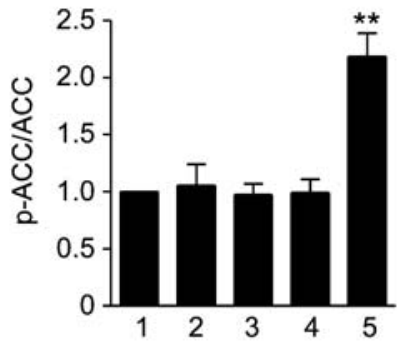

g

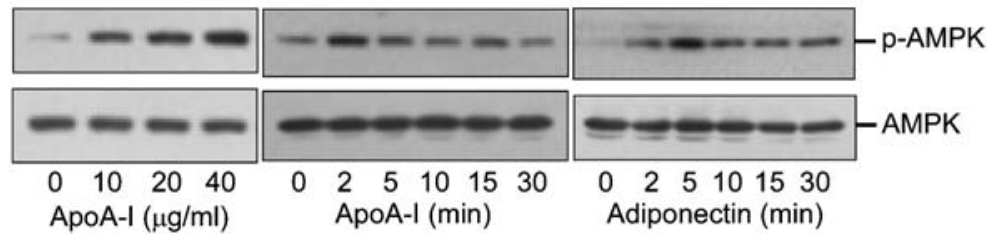

Fig. 1 Effect of APOA-I on AMPK and ACC activation in C2C12 myotubes. a-c Effect of APOA-I on AMPK and ACC phosphorylation. Differentiated $\mathrm{C} 2 \mathrm{C} 12$ myocytes were serum-starved for $3 \mathrm{~h}$ and treated with or without $10 \mu \mathrm{g} / \mathrm{ml}$ full-length adiponectin, $2.5 \mu \mathrm{g} / \mathrm{ml}$ globular adiponectin (gAdiponectin), and $20 \mu \mathrm{g} / \mathrm{ml}$ APOA-I for 10 min. Total AMPK, phosphorylated (p-) AMPK, total ACC and pACC were detected by western blotting with specific antibodies. b, c Graphic representation of the relative phosphorylation levels of AMPK and ACC is also shown, with the error bars representing means $\pm \mathrm{SD}$ from three independent experiments. ${ }^{* *} p<0.01$ vs the untreated group. $\mathbf{d}-\mathbf{f}$ Using the same experimental procedures as in $\mathbf{a}$

we found that AMPK phosphorylation was not affected by BSA $(20 \mu \mathrm{g} / \mathrm{ml}), \mathrm{M}-\beta \mathrm{CD}(2.5 \mathrm{mmol} / \mathrm{l})$ or APOB $(20 \mu \mathrm{g} / \mathrm{ml})$. e, f Graphic representation of the relative phosphorylation levels of AMPK and $\mathrm{ACC}$, with the error bars representing means $\pm \mathrm{SD} .{ }^{* *} p<0.01$ vs the untreated group. $g$ The dose- and time-dependent effect of APOA-I on AMPK phosphorylation. Differentiated $\mathrm{C} 2 \mathrm{C} 12$ cells were serumstarved and treated with different concentrations of APOA-I for $10 \mathrm{~min}$, with $20 \mu \mathrm{g} / \mathrm{ml} \mathrm{APOA}-\mathrm{I}$ for various lengths of time, or with $10 \mu \mathrm{g} / \mathrm{ml}$ full-length adiponectin for various lengths of time. Total AMPK and p-AMPK were analysed by immunoblotting

epitope Alexa Fluor 555. When differentiated $\mathrm{C} 2 \mathrm{C} 12$ cells were incubated with the fluorescence-labelled APOA-I, endocytosis of APOA-I was clearly seen in the cells at $2 \mathrm{~h}$ after the start of incubation (Fig. 3a), providing convincing evidence that $\mathrm{C} 2 \mathrm{C} 12$ myotubes are able to uptake APOA-I. We next analysed the molecular mechanism underlying endocytosis of APOA-I. C2C12 cells were simultaneously incubated with fluorescence-labelled APOA-I (red) and Alexa Fluor 488- labelled transferrin (green), a molecular marker of the recycling endosome. It appeared that the majority of internalised APOA-I was colocalised with transferrin (Fig. 3b). These data suggest that APOA-I could be internalised into $\mathrm{C} 2 \mathrm{C} 12$ myotubes through a clathrindependent endocytotic process, and that the internalised APOA-I is sorted into the endosomal recycling compartment. To analyse whether APOA-I is internalised in a 
Fig. 2 Effect of APOA-I on glucose uptake in $\mathrm{C} 2 \mathrm{C} 12$ myotubes. Differentiated C2C12 cells were serum-starved for $3 \mathrm{~h}$ and treated with full-length adiponectin $(25 \mu \mathrm{g} / \mathrm{ml})$, APOA-I $(20 \mu \mathrm{g} / \mathrm{ml})$ or insulin $(1 \mu \mathrm{mol} / \mathrm{l})$ for $30 \mathrm{~min}$, followed by a glucose-uptake assay. a The effect of APOA-I and adiponectin on glucose uptake in $\mathrm{C} 2 \mathrm{C} 12$ myocytes. b The effect of APOA-I and insulin on glucose uptake in $\mathrm{C} 2 \mathrm{C} 12$ myocytes. c The dose-dependent effect of APOA-I. The error bars represent the means \pm SD of three independent experiments. $* p<0.05, * * p<0.01$ vs the untreated group; $* * * p<0.01$ in pair-wise comparisons a

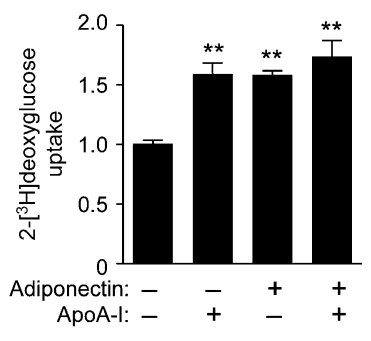

b
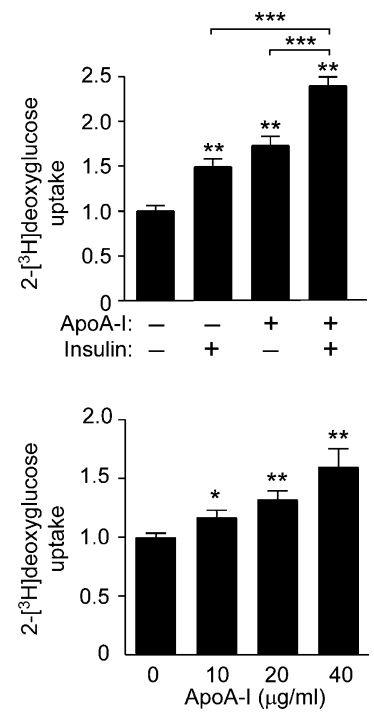

caveolin-dependent manner, C2C12 cells were incubated with fluorescence-labelled APOA-I and stained with an antibody against caveolin-1, followed by fluorescent staining. As shown in Fig. 3c, there was little colocalisation of caveolin-1 with internalised APOA-I, indicating that caveolin-dependent endocytosis is not likely involved in APOA-I uptake into $\mathrm{C} 2 \mathrm{C} 12$ cells.

APOA-I-mediated regulation of AMPK and ACC phosphorylation is dependent on endocytosis Our observation suggests that a clathrin-dependent pathway is involved in the uptake of APOA-I into $\mathrm{C} 2 \mathrm{C} 12$ myotubes. To determine whether endocytosis of APOA-I is required for downstream signalling, we analysed the effect of several endocytosis inhibitors on AMPK activation by APOA-I. Potassium depletion was used to block clathrin-coated pit formation [20]. M- $\beta C D$, which selectively extracts cholesterol from the plasma membrane, was used to prevent caveolaemediated endocytosis [16]. As shown in Fig. 3d-f, potassium depletion strongly suppressed APOA-I-mediated phosphorylation of AMPK and ACC. However, M- $\beta C D$ had a minimal effect on AMPK and ACC activation by APOA-I. Taken together, these data indicate that endocytosis of APOA-I is required for APOA-I-induced AMPK and ACC activation. Furthermore, they suggest that the clathrin-dependent pathway is involved in the endocytosis

of APOA-I, consistent with the observation that the internalised APOA-I was colocalised with transferrin in the recycling endosome (Fig. 3b).

In vivo effects of APOA-I on AMPK phosphorylation and gluconeogenesis AMPK functions as a 'fuel gauge' that plays a central role in monitoring cellular energy status [21-23]. Our observation suggests that endocytosed APOA-I is able to stimulate AMPK in C2C12 myotubes. To provide evidence that APOA-I is involved in the regulation of AMPK activation in vivo, we investigated whether APOA-I could stimulate AMPK phosphorylation in isolated skeletal muscles in the mouse. As shown in Fig. 4a, phosphorylation of AMPK in the soleus muscle was significantly increased after incubation with APOA-I. We also analysed the phosphorylation status of AMPK in skeletal muscle and liver from either wild-type or Apoa- $\Gamma^{1-}$ mice [14]. As expected, phosphorylation of AMPK in soleus muscle and liver was significantly reduced in Apoa-I deficient mice (Fig. 4b,c). These data therefore indicate that endogenous APOA-I is able to positively regulate the in vivo phosphorylation level of AMPK, consistent with our observation that APOA-I can stimulate AMPK phosphorylation in C2C12 myotubes (Fig. 1).

Activation of AMPK has been associated with inhibition of gluconeogenesis in hepatocytes via negative regulation of genes encoding critical enzymes, including phosphoenolpyruvate carboxykinase $(P E P C K)$ and glucose-6-phosphatase (G6Pase) [24]. To determine the potential role of endogenous APOA-I on gluconeogenesis, we analysed the Pepck and G6pase mRNA levels in Apoa- $\Gamma^{\prime-}$ mice by realtime RT-PCR. As shown in Fig. 5, the Pepck and G6Pase mRNA levels were significantly elevated in the livers of Apoa-I-deficient mice compared with those in wild-type mice, implicating endogenous APOA-I in negative regulation of gluconeogenic enzymes. Taken together, these observations indicate that APOA-I is able to modulate critical enzymes involved in the in vivo regulation of glucose utilisation and energy homeostasis.

Role of endogenous APOA-I on energy and glucose homeostasis We next analysed the energy and glucose homeostasis in the Apoa- $\Gamma^{--}$mice. Compared with the wild-type mice, Apoa- $\Gamma^{--}$mice showed no change in body weight for up to 15 weeks on a normal diet (Fig. 6a). There was no difference in food intake between $\mathrm{Apoa}-\Gamma^{\prime-}$ mice and wild-type mice $(2.69 \pm 0.32 \mathrm{~g}$ /day in wild-type mice vs $2.66 \pm 0.36 \mathrm{~g} /$ day in Apoa- $\Gamma^{/-}$mice; Fig. 6b). However, body fat content was significantly increased in Apoa- $\Gamma^{1-}$ mice $(8.97 \pm 1.6 \%$ of fat/body in wild-type mice vs $14.6 \pm 2.1 \%$ in $A$ poa $-\Gamma^{--}$mice; Fig. 6c). Consistent with this observation, retroperitoneal fat pad weight was significantly higher in Apoa- $\Gamma^{/-}$mice than in the wild-type animals (Fig. 6d). 
Fig. 3 Endocytosis of APOA-I and the effect of endocytosis on AMPK and ACC phosphorylation. a Endocytosis of APOA-I. Differentiated $\mathrm{C} 2 \mathrm{C} 12$ cells were incubated with Alexa Fluor 555 labelled APOA-I (red) at $37^{\circ} \mathrm{C}$ for $2 \mathrm{~h}$. The cells were washed, fixed and analysed by confocal microscopy. The cell perimeter is marked by dotted lines. $\mathbf{b}$ Colocalisation of APOA-I with transferrin. $\mathrm{C} 2 \mathrm{C} 12$ myotubes were serum-starved for $2 \mathrm{~h}$ and then incubated with Alexa Fluor 488-conjugated transferrin (green) and Alexa Fluor 555labelled APOA-I (red) for $2 \mathrm{~h}$, followed by confocal microscopy analysis. c Colocalisation studies of APOA-I with caveolin. $\mathrm{C} 2 \mathrm{C} 12$ cells were serum-starved for $2 \mathrm{~h}$ and then incubated with Alexa 555labelled APOA-I (red) for $2 \mathrm{~h}$. The cells were fixed and immunostained with an anti-caveolin antibody followed by Alexa 488-conjugated secondary antibodies (green). d-f Roles of endocytosis inhibitors on APOA-I-activated AMPK and ACC phosphorylation. C2C12 cells were serum-starved for $2 \mathrm{~h}$, incubated with $\mathrm{K}^{+}$depletion buffer or $\mathrm{M}-\beta \mathrm{CD}$ at $37^{\circ} \mathrm{C}$ for $1 \mathrm{~h}$. The cells were treated with $20 \mu \mathrm{g} / \mathrm{ml}$ APOA-I for $10 \mathrm{~min}$. Phosphorylation and the total amount of AMPK and ACC were detected by western blotting with specific antibodies, as indicated (d). e, f Graphic representation of the relative phosphorylation levels of AMPK and ACC. The results are presented as the means $\pm \mathrm{SD}$ of three independent experiments. ${ }^{* *} p<0.01$ vs the untreated group a
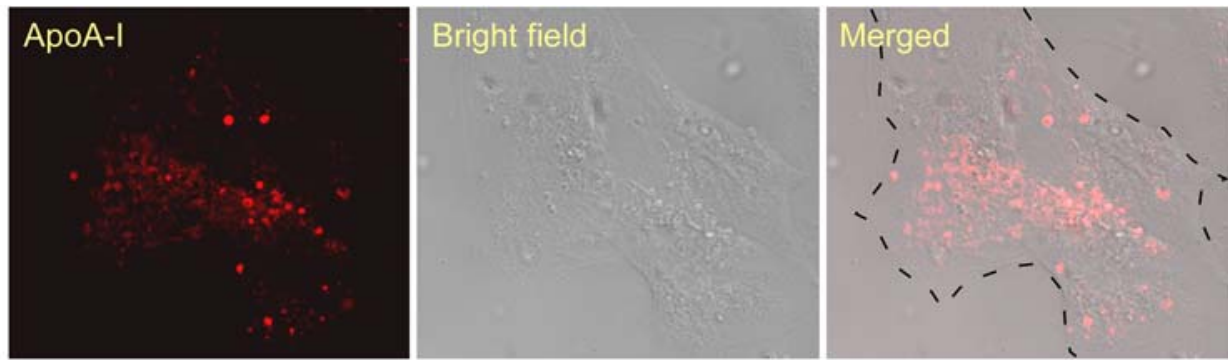

b
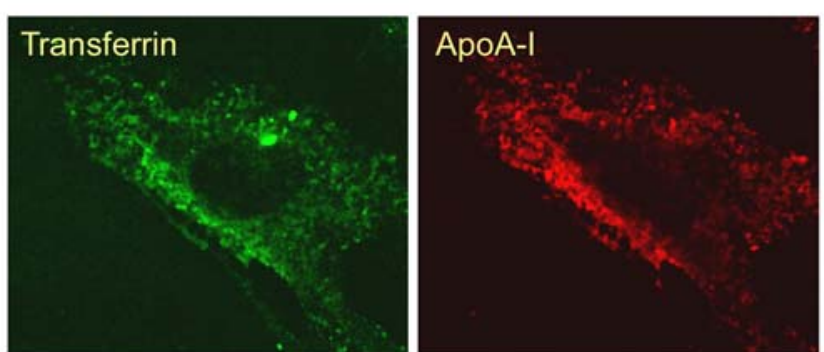

Merged

C
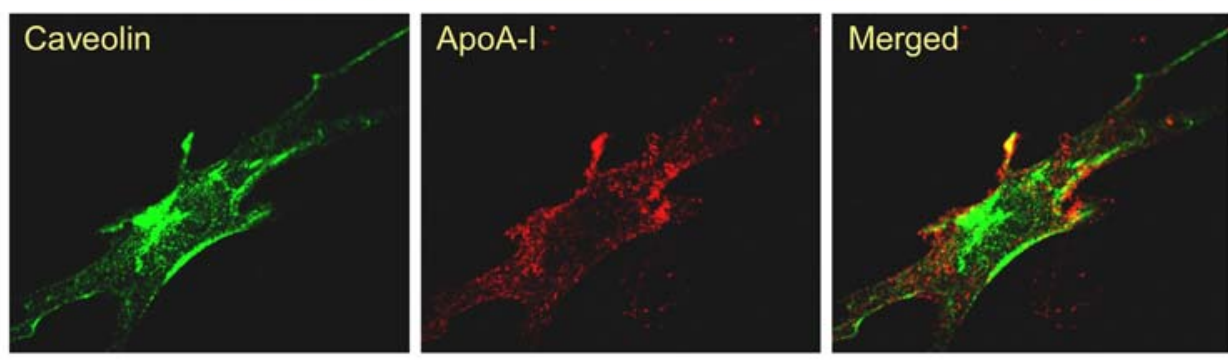

d
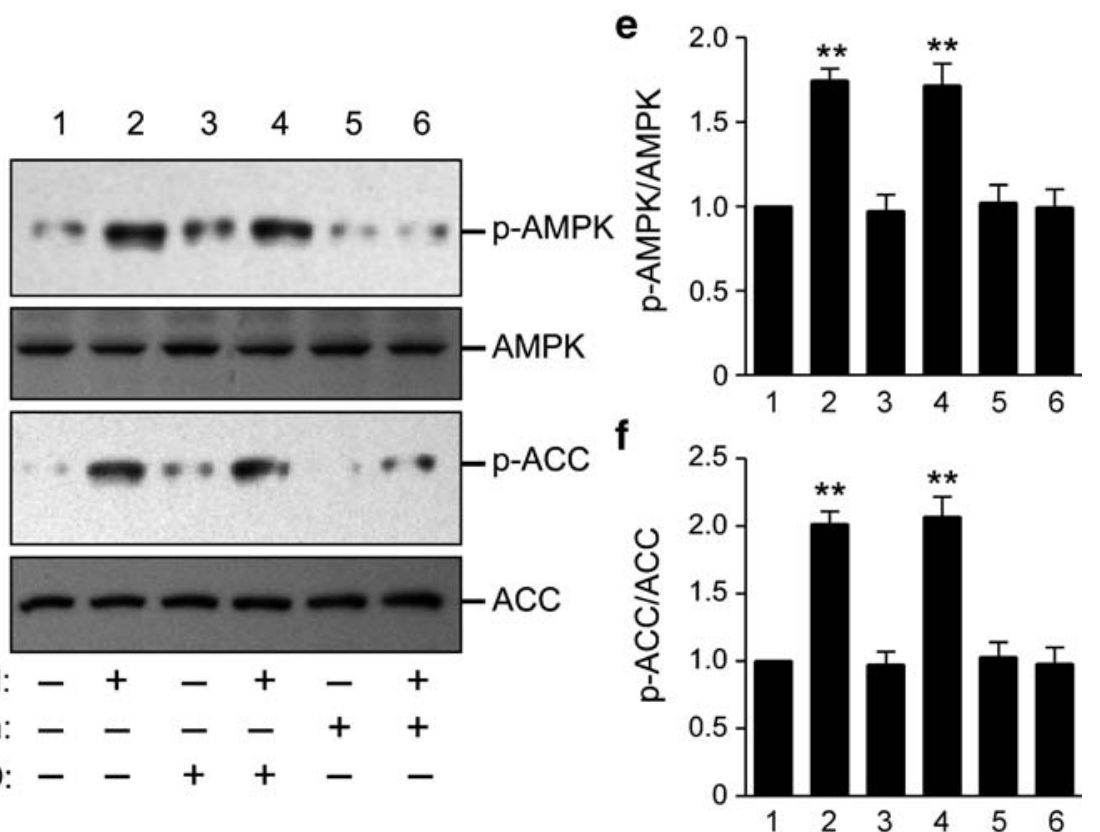

To evaluate the potential role of APOA-I in glucose and insulin homeostasis, blood glucose and insulin levels were measured in animals fasted for $3 \mathrm{~h}$. The plasma glucose level was significantly increased in Apoa- $\Gamma^{1-}$ mice (Fig. 7a), and this was accompanied by an increase in serum insulin (Fig. 7b), indicating that $A p o a-\Gamma^{-1}$ mice show signs of hyperglycaemia and hyperinsulinaemia. We next performed a glucose tolerance test on the animals. The plasma glucose concentrations was significantly increased in Apoa $-\Gamma^{1-}$ mice following glucose administration 
a

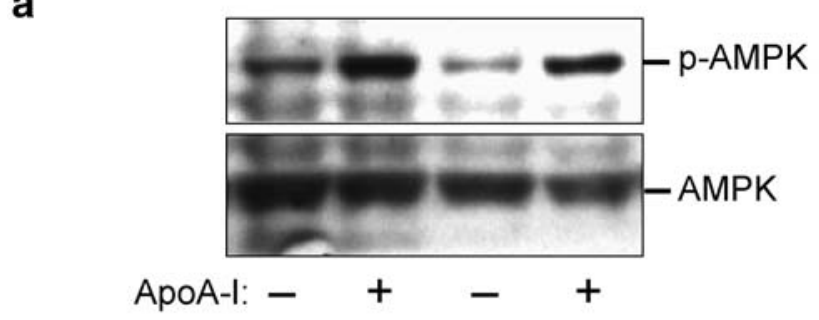

b

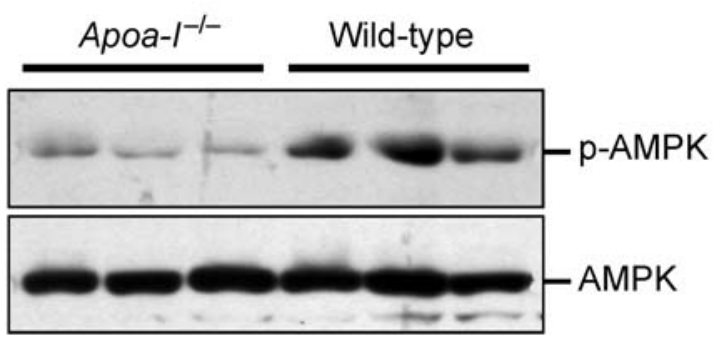

C

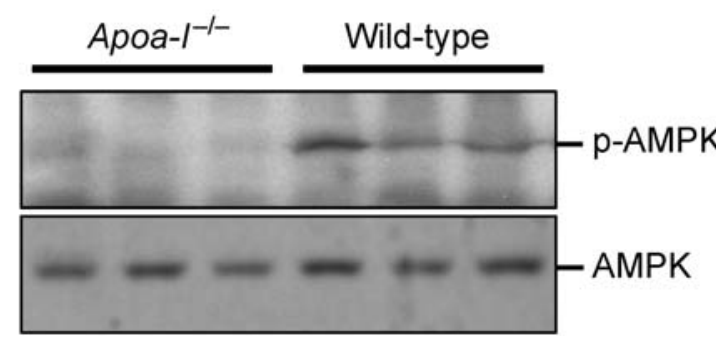

Fig. 4 In vivo effect of APOA-I on AMPK phosphorylation. (a) AMPK phosphorylation was analysed with isolated soleus muscle from wide type mice fasted for $3 \mathrm{~h}$. The skeletal muscle tissues were incubated with APOA-I $(40 \mu \mathrm{g} / \mathrm{ml})$ for $20 \mathrm{~min}$ and used in western blotting analysis. (b) Levels of phosphorylated (p-) AMPK were determined in soleus muscle and liver from overnight-fasted Apoa- $\Gamma^{1-}$ mice and control mice at 12 weeks of age

(Fig. 6c). In line with this, the AUC was significantly increased in these mice (Fig. 6c). These results indicate that the Apoa- $\Gamma^{--}$mice had a slower glucose clearance than the wild-type animals. Collectively, our data suggest that a lack of APOA-I is associated with impaired glucose homeostasis
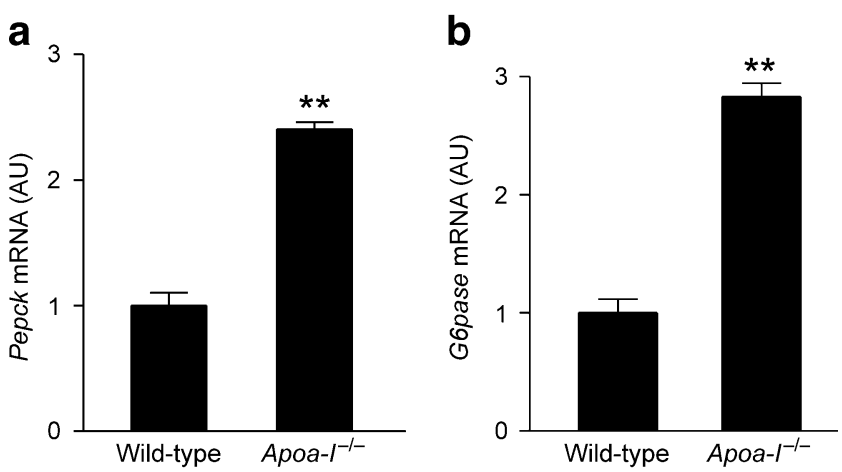

Fig. 5 In vivo effect of APOA-I on the expression of gluconeogenic enzymes. Pepck (a) and G6Pase (b) mRNA levels were determined by real-time RT-PCR using liver RNA isolated from overnight-fasted mice, and normalised with the level of $\beta$-actin mRNA. There were three mice in each experimental group, and data are shown as means \pm SD. ${ }^{* *} p<0.01$ vs wild-type

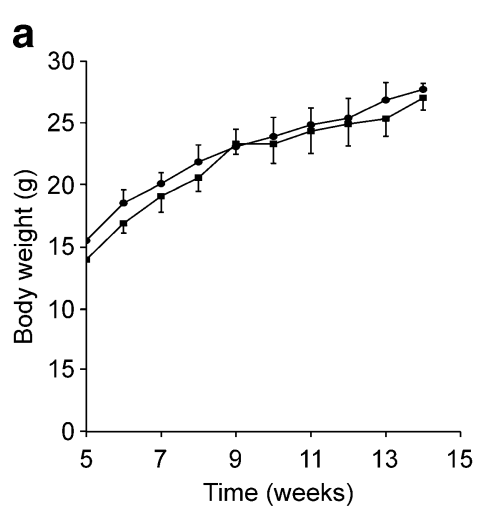

b

C

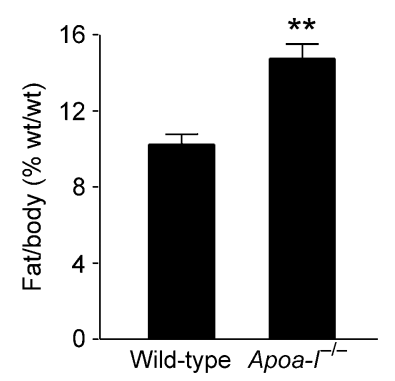

d

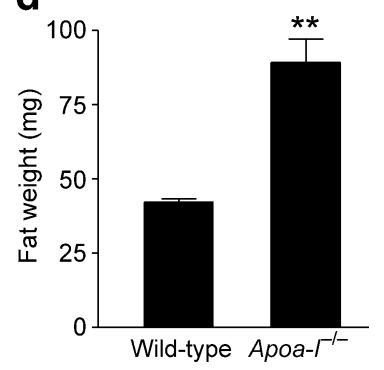

Fig. 6 Increased fat mass in Apoa- $\Gamma^{/-}$mice. The growth curve (a), food intake (b) and fat content (c) were determined for male Apoa- $\Gamma^{-1}$ and wild-type mice ( $n=5$ for each group) at 15 weeks. $\mathbf{d}$ The weight of the retroperitoneal fat pad was measured in 12-week-old male mice $(n=7$ for Apoa- $\Gamma^{/-}$mice, $n=8$ for wild-type mice). ${ }^{* *} p<0.01$ vs wild-type

in vivo, further supporting our observation that APOA-I modulates energy and glucose metabolism through activation of AMPK.

\section{Discussion}

In humans, one of the hallmarks of insulin resistance and the metabolic syndrome is a decreased plasma concentration of HDL and its major component, APOA-I [6-9]. It has long been postulated that APOA-I and HDL levels are inversely associated with the incidence of the metabolic syndrome [7, 8], which is associated with an excess risk of type 2 diabetes and cardiovascular disease. In this report, we provide both in vitro and in vivo evidence implicating APOA-I in the maintenance of glucose homeostasis via activation of AMPK. We report the following observations: (1) APOA-I stimulates phosphorylation of AMPK and ACC and elevates glucose uptake in $\mathrm{C} 2 \mathrm{C} 12$ myocytes; (2) APOA-I can be internalised into $\mathrm{C} 2 \mathrm{C} 12$ myotubes in an endosomal recycling compartment; (3) APOA-I-mediated stimulation of AMPK in $\mathrm{C} 2 \mathrm{C} 12$ cells is dependent on endocytosis, as inhibition of clathrin-dependent endocytosis blunted the action of APOA-I; (4) APOA-I is able to stimulate AMPK phosphorylation in vivo using isolated skeletal muscle from the mice; (5) Apoa-I deletion in the mouse is associated with a reduction in AMPK phosphor- 
a

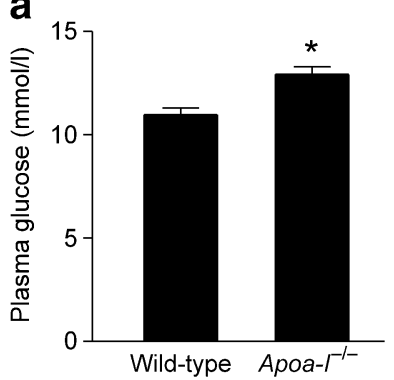

b

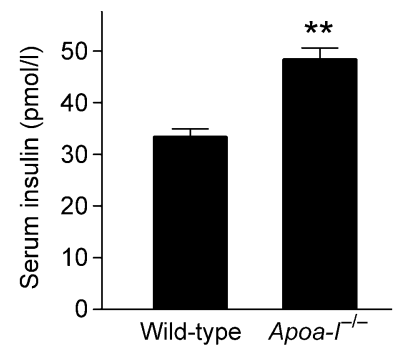

C

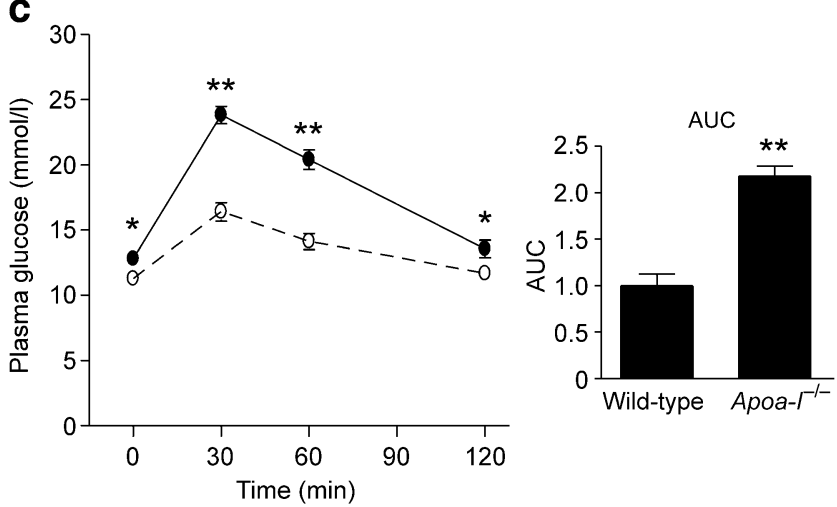

Fig. 7 Analysis of energy and glucose homeostasis in Apoa- $\Gamma^{\prime-}$ mice. At 12 weeks of age, the plasma glucose concentrations (a) and serum insulin concentrations (b) were determined in male Apoa- $\Gamma^{1-}$ mice and wild-type mice fasted for $3 \mathrm{~h}$ ( $n=7$ for Apoa- $\Gamma^{/-}$mice, $n=8$ for wild-type mice). c For the glucose tolerance test, the blood glucose levels were determined for the mice at the indicated time points following the intraperitoneal administration of glucose. $\mathbf{d}$ The glucose AUC during the tolerance test. ${ }^{*} p<0.05,{ }^{* *} p<0.01$ vs wild-type

ylation in liver and skeletal muscle, and an increase in the expression of gluconeogenic enzymes in liver; and (6) Apoa- $\Gamma^{/-}$mice had an increased fat content and a compromised glucose tolerance test, indicative of impaired glucose homeostasis.

Our findings, which suggest that APOA-I is involved in the modulation of glucose metabolism, are supported by recent studies with mouse models reporting associations between a reduced function or amount of APOA-I and compromised glucose homeostasis. ABCA1 is one of the most important binding proteins for plasma APOA-I [3]. $A B C A 1$ deletion in the mouse leads to IGT [25], similar to our findings with the Apoa- $\Gamma^{/-}$mouse. Apolipoprotein A-II (APOA-II) is the second most abundant protein in HDL. In Apoa-II transgenic mice, the level of APOA-I in HDL was dramatically reduced [26]. ApoA-II transgenic mice have increased adipose mass, fasting plasma insulin and insulin resistance [27]. This is consistent with our findings revealing that APOA-I positively modulates glucose metabolism. Furthermore, the modulatory function of APOA-I in glucose homeostasis is supported by the finding that the mutated AA genotype of the Apoa-I MspI polymorphism is associated with an increased risk of IGT and type 2 diabetes in human subjects [12].
In our experiment, we observed that APOA-I is able to stimulate phosphorylation of AMPK and ACC, and elevates glucose uptake in $\mathrm{C} 2 \mathrm{C} 12$ myocytes, consistent with a recent report showing that APOA-I stimulates AMPK phosphorylation in endothelial cells by interacting with eNOS after endocytosis [5]. In our studies, selectively extracting cholesterol from the plasma membrane using $\mathrm{M}$ $\beta C D$ had no effect on AMPK phosphorylation in $\mathrm{C} 2 \mathrm{C} 12$ myotubes, indicating that cholesterol efflux itself does not affect AMPK phosphorylation in skeletal muscles. Our data suggest that APOA-I could be internalised into $\mathrm{C} 2 \mathrm{C} 12$ myotubes via clathrin-dependent endocytosis. This is consistent with a recent observation demonstrating that HDL could be endocytosed into the transferrin-containing compartment via SR-BII [28]. There are other APOA-I binding proteins $[29,30]$ in addition to ABCA1, SR-BI and SR-BII. Using RT-PCR we found that the mRNA transcripts of the genes encoding SR-BI, SR-BII and ABCA1 are present in $\mathrm{C} 2 \mathrm{C} 12$ myotubes (data not shown). The nature of membrane receptor(s) or other molecules involved in APOA-I endocytosis in skeletal muscle remains to be determined.

Taken together, our study reveals a new paradigm to explain the protective effect of APOA-I in type 2 diabetes and the metabolic syndrome. We propose a model in which the circulating APOA-I, after being internalised through a clathrin-dependent endocytotic process, activates AMPK and affects the energy and glucose metabolism of the cell. The activation of AMPK leads to an increase in glucose uptake in skeletal muscle and a reduction in gluconeogenesis in the liver. Through this mechanism, APOA-I stimulates glucose utilisation and improves insulin resistance in peripheral tissues. In this regard, APOA-I has a dual protective effect in type 2 diabetes, lowering the prevalence of diabetes-associated cardiovascular diseases and improving insulin resistance via activation of AMPK. It can therefore be envisioned that an increase in APOA-I in human patients with type 2 diabetes would improve both insulin resistance and cardiovascular disease. In this regard, APOA-I seems to be an ideal target for the prevention and therapy of type 2 diabetes and the metabolic syndrome.

Acknowledgements We wish to thank Y. Liu and L. Dong for technical assistance. This work was supported by research grants from Chinese Academy of Sciences (One Hundred Talents Program and The Knowledge Innovation Program KSCX1-YW-02), National Natural Science Foundation of China (30588002 and 30470870), Science and Technology Commission of Shanghai Municipality (04dz14007), Ministry of Science and Technology of China (2006CB503900 and 2006CB943902) and 'Western Light' Program of Talent to Y. Chen.

Duality of interest The authors declare that there is no duality of interest associated with this manuscript. 


\section{References}

1. Assmann G, Nofer JR (2003) Atheroprotective effects of highdensity lipoproteins. Annu Rev Med 54:321-341

2. Linsel-Nitschke P, Tall AR (2005) HDL as a target in the treatment of atherosclerotic cardiovascular disease. Nat Rev Drug Discov 4:193-205

3. Oram JF, Heinecke JW (2005) ATP-binding cassette transporter A1: a cell cholesterol exporter that protects against cardiovascular disease. Physiol Rev 85:1343-1372

4. Silver DL, Tall AR (2001) The cellular biology of scavenger receptor class B type I. Curr Opin Lipidol 12:497-504

5. Drew BG, Fidge NH, Gallon-Beaumier G, Kemp BE, Kingwell BA (2004) High-density lipoprotein and apolipoprotein AI increase endothelial NO synthase activity by protein association and multisite phosphorylation. Proc Natl Acad Sci USA 101:6999-7004

6. Reaven GM (1988) Banting lecture 1988. Role of insulin resistance in human disease. Diabetes 37:1595-1607

7. Eckel RH, Grundy SM, Zimmet PZ (2005) The metabolic syndrome. Lancet 365:1415-1428

8. Dandona P, Aljada A, Chaudhuri A, Mohanty P, Garg R (2005) Metabolic syndrome: a comprehensive perspective based on interactions between obesity, diabetes, and inflammation. Circulation 111:1448-1454

9. Mooradian AD, Haas MJ, Wong NC (2004) Transcriptional control of apolipoprotein A-I gene expression in diabetes. Diabetes 53:513-520

10. Frenais R, Ouguerram K, Maugeais C et al (1997) High density lipoprotein apolipoprotein AI kinetics in NIDDM: a stable isotope study. Diabetologia 40:578-583

11. Murao K, Wada Y, Nakamura T, Taylor AH, Mooradian AD, Wong NC (1998) Effects of glucose and insulin on rat apolipoprotein A-I gene expression. J Biol Chem 273:18959-18965

12. Morcillo S, Cardona F, Rojo-Martinez G et al (2005) Association between MspI polymorphism of the APO AI gene and type 2 diabetes mellitus. Diabet Med 22:782-788

13. Klip A, Guma A, Ramlal T, Bilan PJ, Lam L, Leiter LA (1992) Stimulation of hexose transport by metformin in L6 muscle cells in culture. Endocrinology 130:2535-2544

14. Williamson R, Lee D, Hagaman J, Maeda N (1992) Marked reduction of high density lipoprotein cholesterol in mice genetically modified to lack apolipoprotein A-I. Proc Natl Acad Sci USA 89:7134-7138

15. Kadowaki T, Yamauchi T (2005) Adiponectin and adiponectin receptors. Endocr Rev 26:439-451

16. Le PU, Benlimame N, Lagana A, Raz A, Nabi IR (2000) Clathrinmediated endocytosis and recycling of autocrine motility factor receptor to fibronectin fibrils is a limiting factor for NIH-3T3 cell motility. J Cell Sci 113:3227-3240

17. Yamauchi T, Kamon J, Minokoshi Y et al (2002) Adiponectin stimulates glucose utilization and fatty-acid oxidation by activating AMP-activated protein kinase. Nat Med 8:1288-1295

18. Tomas E, Tsao TS, Saha AK et al (2002) Enhanced muscle fat oxidation and glucose transport by ACRP30 globular domain: acetyl-CoA carboxylase inhibition and AMP-activated protein kinase activation. Proc Natl Acad Sci USA 99:16309-16313

19. Wu X, Motoshima H, Mahadev K, Stalker TJ, Scalia R, Goldstein BJ (2003) Involvement of AMP-activated protein kinase in glucose uptake stimulated by the globular domain of adiponectin in primary rat adipocytes. Diabetes 52:1355-1363

20. Larkin JM, Brown MS, Goldstein JL, Anderson RG (1983) Depletion of intracellular potassium arrests coated pit formation and receptor-mediated endocytosis in fibroblasts. Cell 33:273-285

21. Kahn BB, Alquier T, Carling D, Hardie DG (2005) AMPactivated protein kinase: ancient energy gauge provides clues to modern understanding of metabolism. Cell Metab 1:15-25

22. Carling D (2004) The AMP-activated protein kinase cascade - a unifying system for energy control. Trends Biochem Sci 29:18-24

23. Musi N (2006) AMP-activated protein kinase and type 2 diabetes. Curr Med Chem 13:583-589

24. Lochhead PA, Salt IP, Walker KS, Hardie DG, Sutherland C (2000) 5-aminoimidazole-4-carboxamide riboside mimics the effects of insulin on the expression of the 2 key gluconeogenic genes PEPCK and glucose-6-phosphatase. Diabetes 49:896-903

25. Brunham LR, Kruit JK, Pape TD et al (2007) Beta-cell ABCA1 influences insulin secretion, glucose homeostasis and response to thiazolidinedione treatment. Nat Med 13:340-347

26. de Beer MC, Castellani LW, Cai L, Stromberg AJ, de Beer FC, van der Westhuyzen DR (2004) ApoA-II modulates the association of HDL with class B scavenger receptors SR-BI and CD36. J Lipid Res 45:706-715

27. Castellani LW, Goto AM, Lusis AJ (2001) Studies with apolipoprotein A-II transgenic mice indicate a role for HDLs in adiposity and insulin resistance. Diabetes 50:643-651

28. Eckhardt ER, Cai L, Sun B, Webb NR, van der Westhuyzen DR (2004) High density lipoprotein uptake by scavenger receptor SRBII. J Biol Chem 279:14372-14381

29. Martinez LO, Jacquet S, Esteve JP et al (2003) Ectopic beta-chain of ATP synthase is an apolipoprotein A-I receptor in hepatic HDL endocytosis. Nature 421:75-79

30. Kozyraki R, Fyfe J, Kristiansen M et al (1999) The intrinsic factor-vitamin B12 receptor, cubilin, is a high-affinity apolipoprotein A-I receptor facilitating endocytosis of high-density lipoprotein. Nat Med 5:656-661 